\title{
REPOSITÓRIO DIGITAL
}

\section{UNIVERSIDADE FEDERAL DO RIO GRANDE DO SUL}

\section{S \\ UFRGS}

Porto Alegre, RS - Brasil 


\section{LUME}

Repositório Digital da Universidade Federal do Rio Grande do Sul

$\Leftrightarrow$ Implementado em janeiro de 2008, a partir da proposta de ampliação da Biblioteca Digital de Teses e Dissertações (BD-UFRGS) 


\section{OBJETIVOS}

Reunir, preservar, divulgar e garantir o acesso permanente aos documentos acadêmicos, científicos e artísticos gerados na Universidade, bem como às suas coleções históricas e a outros documentos de relevância para a Instituição, que fazem parte de suas coleções, embora não produzidos por ela. 


\section{REGULAMENTAÇÃO}

> Portaria n 1774 de 03/06/2005 (Reitoria)

Cria a BD-UFRGS

D Resolução n 129/2005 de 12/07/2005 (Câmara de Pós-Graduação)

Estabelece a obrigatoriedade de entrega da tese, dissertação ou trabalho de conclusão de mestrado gerado na UFRGS na forma impressa e em meio eletrônico às bibliotecas. 


\section{REGULAMENTAÇÃO}

D Portaria n 3805 de 20/07/2009 (Reitoria)

Designa o Comitê Gestor do LUME, integrado por docentes representantes das grandes áreas do conhecimento e da Biblioteca Central, Centro de Processamento de Dados e Secretaria de Ensino a Distância 


\section{Cabe ao Comitê Gestor definir a}

\section{política institucional de informação}

para o LUME 
Cada comunidade possui fluxos de informação e metadados definidos conforme suas características e particularidades

Os órgãos envolvidos e suas responsabilidades em cada uma das etapas de inclusão do objeto digital no LUME depende do tipo de documento.

Ex. Teses e Dissertações

- Autor

- Secretaria do Programa de Pós-Graduação

- Biblioteca setorial

- Centro de Processamento de Dados 


\section{SOFTWARES UTILIZADOS}

- DSPACE - Institutional Digital Repository System (MIT e HP)

- instalado em ambiente Linux

- desenvolvido em linguagem Java

- customizável

- utiliza ferramentas de código aberto:

$\rightarrow$ BD Postgre SQL

$\rightarrow$ Servidor de aplicações TOMCAT

$\rightarrow$ Motor de pesquisa LUCÈNE

$\rightarrow$ Servidor Web Apache 


\section{SOFTWARES}

- MANAKIN (Texas A\&M) - customização da interface gráfica filosofia orientada a aspectos

- HANDLE SYSTEM (CNRI) - identificador persistente

- SAMBA - compartilhamento de arquivos em rede

- SSL - autenticação de documentos digitais de acesso restrito 


\section{AMBIENTE}

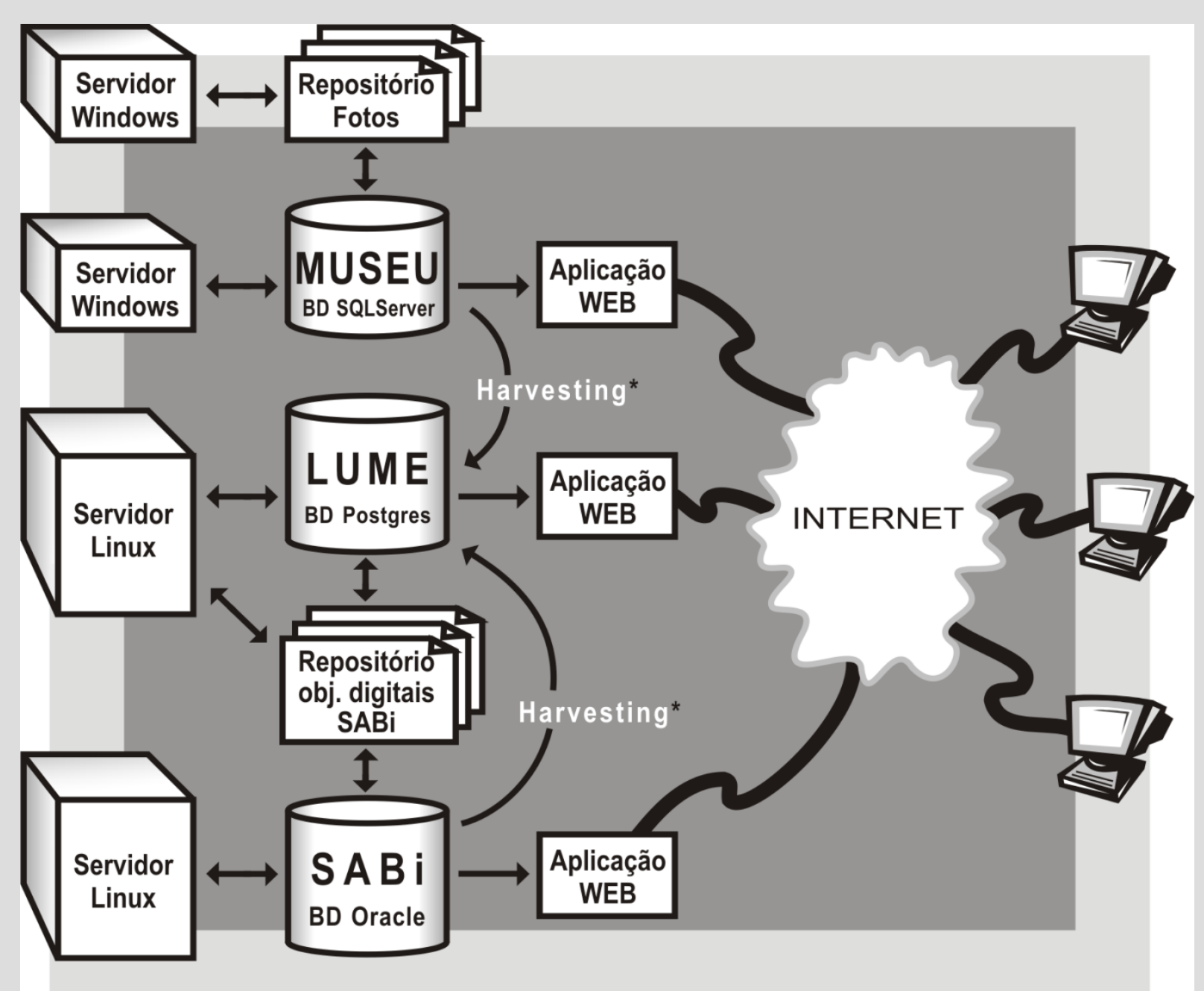

*harvesting incremental: coleta automática de metadados a cada 24 horas.

Figura 1 - Arquitetura do LUME. 


\section{ORGANIZAÇÃO}

$>$ Comunidade: política própria de informação, fluxo de depósito e níveis de acesso aos documentos

$>$ Subcomunidade - divisão da comunidade

$>$ Coleção - divisão da comunidade/subcomunidade: composta de itens

$>$ Item: representa o conteúdo digital 


\section{ORGANIZAÇÃO}

Cada item é composto por:

- metadados: elementos com semântica padronizada

- dados sobre dados (título, autor, assunto, ano, etc.)

- utilizam padrão Dublin Core

- garantia de interoperabilidade

- protocolo OAI-PMH (Open Archives Initiative Protocol for Metadata Harvesting)

arquivos digitais (bitstreams) 


\section{ORGANIZAÇÃO}

ELista de comunidades - Windows Internet Explorer

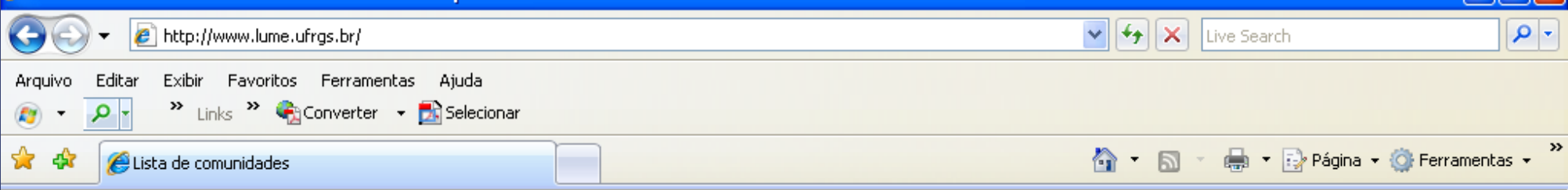

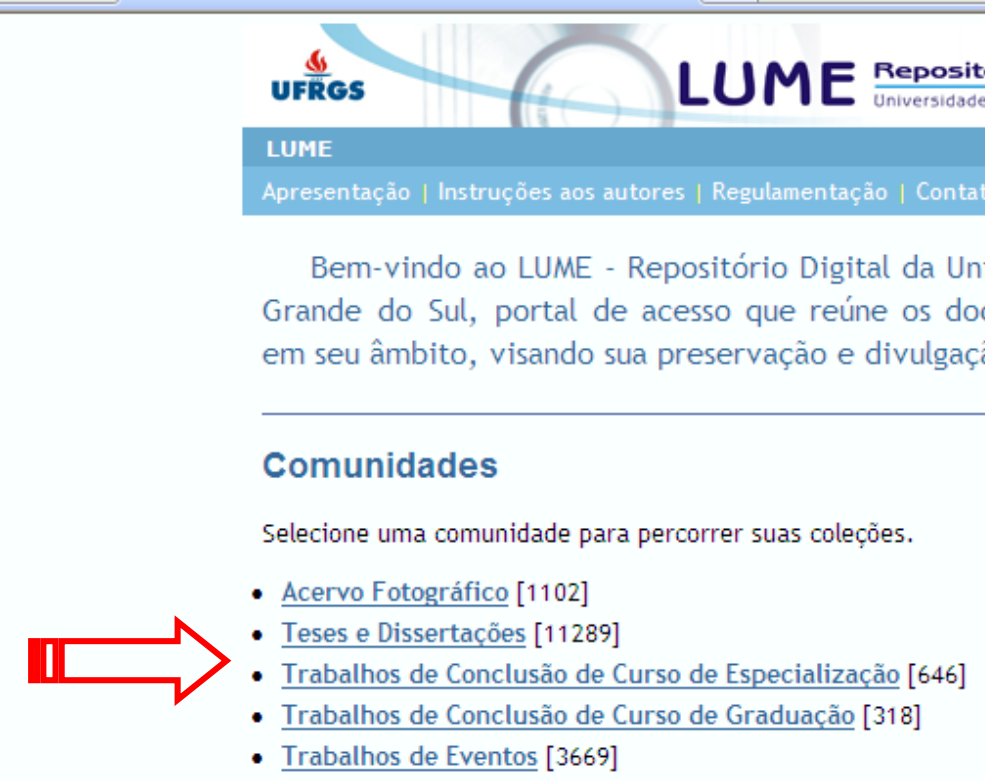

Pesquisar Enviar Pesquisa avancada

Percorrer

Todo o repositório

$\rightarrow$ Comunidades

colecões

$\rightarrow$ Títulos

$\rightarrow$ Autores

$\rightarrow$ Palavras-chave

$\rightarrow$ Anos

Meu cadastro

$\rightarrow$ Login UFRGS

$\rightarrow$ Login não UFRGS

Estatísticas

$\rightarrow$ Downloads

$\rightarrow$ Itens mais

baixados 


\section{ORGANIZAÇÃO}

Permite disponibilizar vários tipos de arquivos:

- Texto

Imagem

> Vídeo

Áudio 


\section{Exibir Favoritos Ferramentas Ajuda}

"Links " GConverter - ES Selecionar

Thatemática e educação sexual: modelagem do fenôm...

ओ -

process, and Mathematical Modeling, viewed as a learning environment the

enriches such experiences. This work has developed three didactic resources: a)

mathematical model for absorption of daily use contraceptives; b) informational

video on the use of contraceptives; c) teaching plan with a didactic sequence.

The experimentation took place in a regular classroom of a state school of Porto

Alegre and in a laboratory environment, with a small group of students from

Colégio de Aplicação of Universidade Federal do Rio Grande do Sul (UFRGS). The

main results show the didactic resource developed fosters opportunities for

students to discuss and better understand their sexuality, explains the

mechanisms of contraceptives, gives students useful mathematical tools for the

understanding other phenomena, promotes an discussion environment, and

supports the logic articulation among different ideas and mathematical concepts,

thus enabling greater meaningfulness to the learning process. The

experimentation carried out also demonstrates the potential of this media to

stimulate the interest and tho discuscion on Sexual Education and Mathematics.

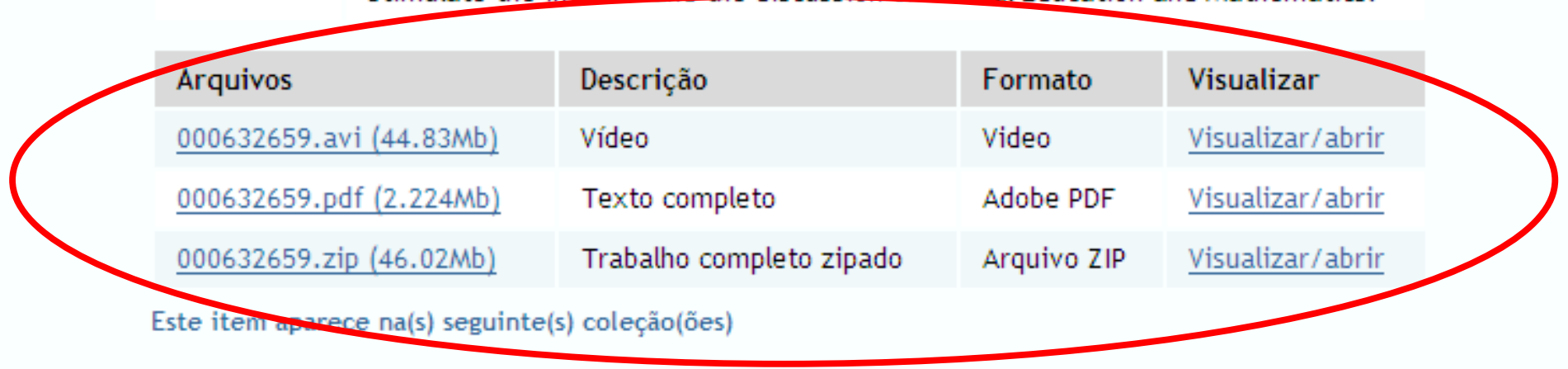

- Ensino de Matemática

Mostrar registro completo

O autor é titular dos direitos autorais dos documentos disponiveis neste repositório e é vedada, nos termos da lei, a comercializaça de qualquer espécie sem sua autorizạ̣ăo prévia. 
LUME

Apresentação | Instruções aos autores | Regulamentação | Contato | Ajuda

\section{Pesquisar Enviar}

Pesquisa avancada

Bem-vindo ao LUME - Repositório Digital da Universidade Federal do Rio Grande do Sul, portal de acesso que reúne os documentos digitais gerados em seu âmbito, visando sua preservação e divulgação.

\section{Comunidades}

Selecione uma comunidade para percorrer suas coleções.

$\longrightarrow$ Acervo Fotográfico [1102]

- Teses e Dissertações [11289]

- Trabalhos de Conclusão de Curso de Especialização [646]

- Trabalhos de Conclusão de Curso de Graduação [318]

- Trabalhos de Eventos [3669]

\section{Percorrer}

\section{Todo o repositório}

$\rightarrow$ Comunidades $e$ colecões

$\rightarrow \underline{\text { Títulos }}$

$\rightarrow$ Autores

$\rightarrow$ Palavras-chave

$\rightarrow$ Anos

Meu cadastro

$\rightarrow$ Login UFRGS

$\rightarrow$ Login não UFRGS

Estatísticas

$\rightarrow$ Downloads

$\rightarrow$ Itens mais baixados 


\section{UFRES LUME $\frac{\mathbb{S}}{\text { Repositório Digital }}$}

LUME $\rightarrow$ Acervo Fotográfico

Apresentação | Instruções aos autores | Regulamentação | Contato | Ajuda

Pesquisar Enviar

\section{Acervo Fotográfico}

Wh Estatísticas

Pesquisar geral

Autor

Título

Palavra-chave

Ano

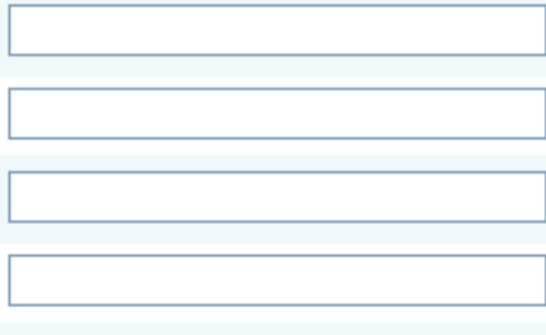

\section{Enviar}

Coleções

Escola de Educação Física [371]

Instituto de Física [258]

Museu Universitário [473]

\section{Entradas recentes}

Inezil Penna Marinho $\mathrm{s} / \mathrm{r}(1947)$
Percorrer

Todo o repositório

$\rightarrow$ Comunidades e colecões

$\rightarrow$ Títulos

$\rightarrow$ Autores

$\rightarrow$ Palavras-chave

$\rightarrow$ Anos

\section{Esta comunidade}

$\rightarrow$ Títulos

$\rightarrow$ Autores

$\rightarrow$ Palavras-chave

$\rightarrow$ Anos

Meu cadastro

$\rightarrow$ Login UFRGS

$\rightarrow$ Login não UFRGS

Estatísticas

$\rightarrow$ Downloads

$\rightarrow$ Itens mais baixados
Pesquisa avancada 


\section{Faculdade de Medicina}

$\underline{\text { Mostrar registro completo }}$

Wh Estatísticas

Para citar ou acessar este item utilize:

\section{http://hdl.handle.net/10183/16282}

Título

Faculdade de Medicina

Fotógrafo

Data de cobertura

\section{Resquim}

anterior a 1965

Envelope original assessoria de Imprensa $n^{\circ} 812$, informações ficha anterior.

Descrição

Palavra-chave

Características do original

URI
Foto da fachada do antigo Prédio da Faculdade de Medicina (1924-1974) abrigou o Instituto de Biociências até 1992, atualmente funciona o Instituto de Ciências Básicas da Saúde.

\section{CAMPUS CENTRAL}

FACULDADE DE MEDICINA INSTITUTO BIOCIÊNCIAS

INSTITUTO DE CIÊNCIAS BÁSICAS DA SAÚDE

foto; réplica; preto e branco; celulóide; dimensões: $12 \mathrm{~cm} \times 18 \mathrm{~cm}$. Estado de conservação: escrita, com manchas

http://hdl.handle.net/10183/16282

\section{Percorrer}

\section{Todo o repositório}

$\rightarrow$ Comunidadese coleções

$\rightarrow$ Títulos

$\rightarrow$ Autores

$\rightarrow$ Palavras-chave

$\rightarrow$ Anos

\section{Esta coleção}

$\rightarrow \underline{\text { Títulos }}$

$\rightarrow$ Autores

$\rightarrow$ Palavras-chave

$\rightarrow$ Anos

Meu cadastro

$\rightarrow$ Login UFRGS

$\rightarrow$ Login não UFRGS

\section{Estatísticas}

$\rightarrow$ Downloads

$\rightarrow$ Itens mais baixados

\begin{tabular}{l|l|l|l|}
\hline Arquivos & Descrição & Formato & Visualizar \\
\hline Rg2040.jpg (115.6Kb) & & \\
\hline
\end{tabular}


ivo Editar Exibir Favoritos Ferramentas Ajuda

- 0 " Links " Q EConverter - Delecionar

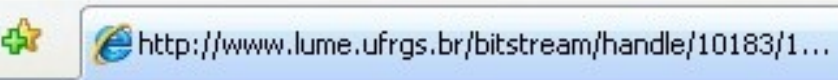

๑)

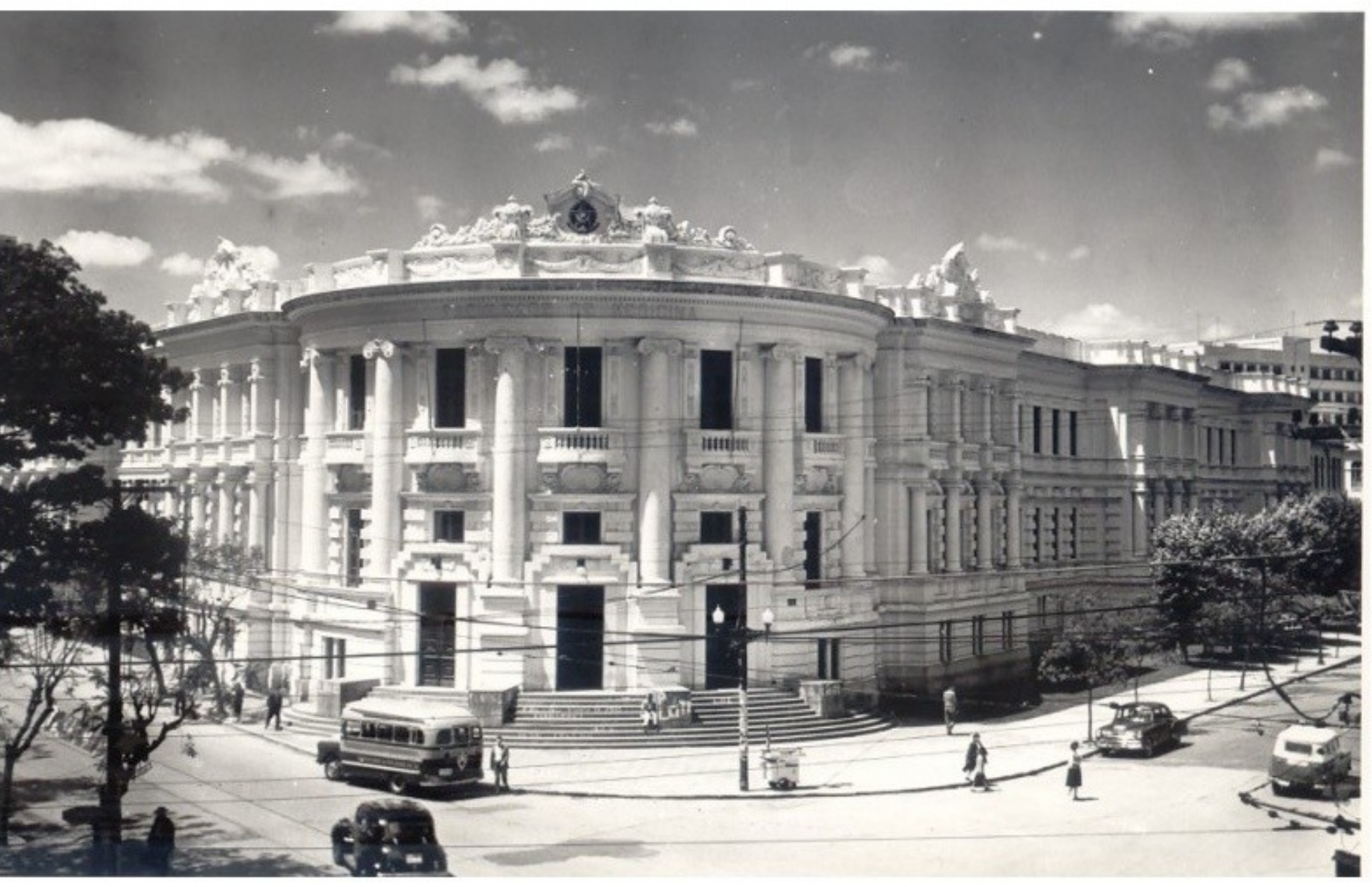




\section{INTERFACE}

ELista de comunidades - Windows Internet Explorer

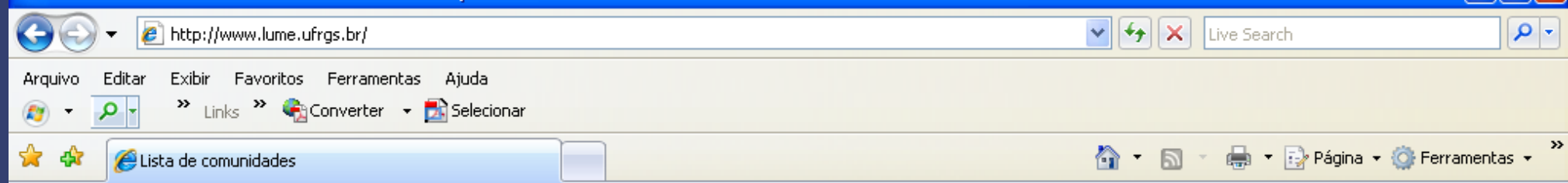

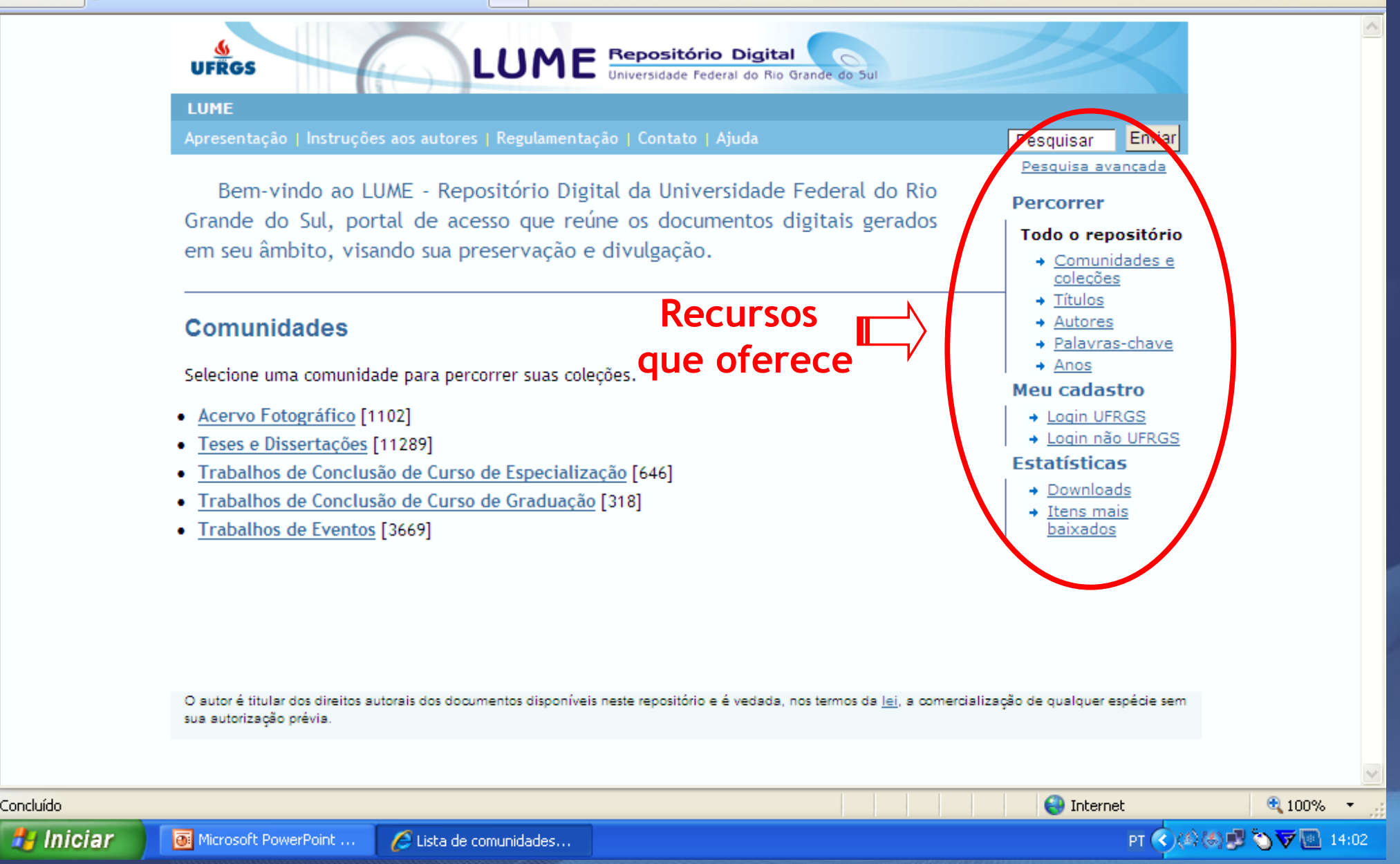




\section{ESTATÍSTICAS}

Implementadas a partir de janeiro de 2008.

Estão presentes em todos os níveis do repositório, da comunidade ao item.

\section{Ll Estatisticas}

Em 23 de outubro foram contabilizados 5.211.720 acessos. 


\section{TIPOS DE ACESSO COLETADOS}

\begin{tabular}{|l|l|}
\hline \multicolumn{1}{|c|}{ Código } & \multicolumn{1}{c}{ Tipo de acesso } \\
\hline 1 & Visualização de item \\
\hline 2 & Visualização de coleção \\
\hline 3 & Visualização de comunidade \\
\hline 4 & Download \\
\hline 5 & Pesquisa filtro \\
\hline 6 & Pesquisa avançada \\
\hline 7 & Pesquisa simples \\
\hline 8 & Percorrer título \\
\hline 9 & Percorrer autor \\
\hline 10 & Percorrer assunto \\
\hline 11 & Percorrer ano \\
\hline 12 & Percorrer itens do autor \\
\hline 13 & Percorrer itens do assunto \\
\hline
\end{tabular}


ค. Mais visitados Guia rápido in Últimas notícias \& Google

\section{LUME Repositbio igital}

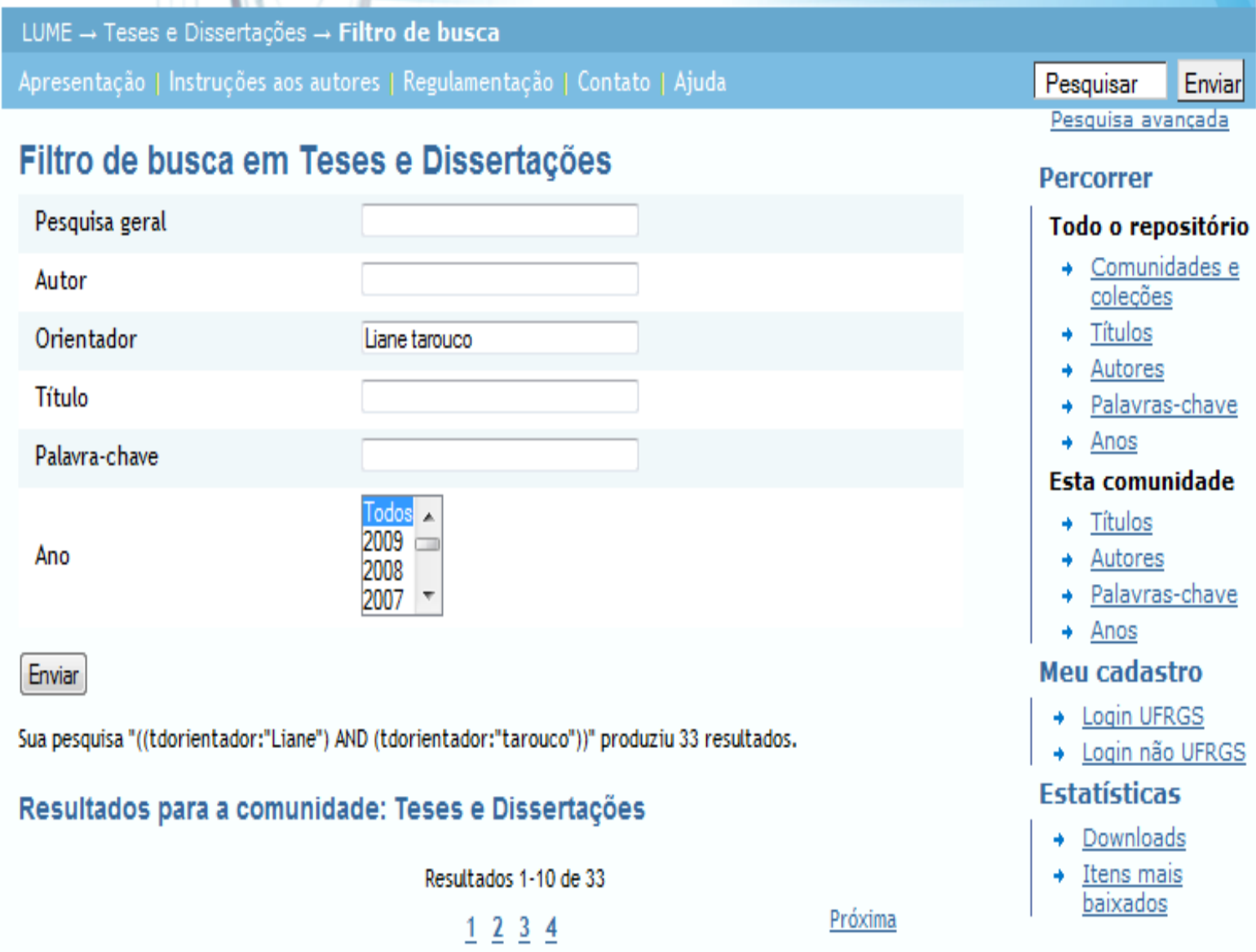

Implementação de um IDS utilizando SNMP e lógica difusa Virti, Émerson Salvadori (2007)

Implementando segurança e controle em redes de computadores Bertholdn I eandro Márcin (1997) 

Arquivo Éditar Exibir Histórico Favoritos Ferramentas Ajụda

. Mais visitados Guia rápido îltimas notícias \& Google

Os usos pedagógicos dos vídeos digit...

\section{( UFR̈GS \\ LUME}

LUME $\rightarrow$ Teses e Dissertações $\rightarrow$ Teses e Dissertações defendidas na UFRGS $\rightarrow$ Multidisciplinar $\rightarrow$ Informática na Educação $\rightarrow$

Visualizar item

Apresentação | Instruções aos autores | Regulamentação | Contato | Ajuda

Pesquisar Enviar

Os usos pedagógicos dos vídeos digitais indexados Pesquisa avancada

Mostrar registro completo

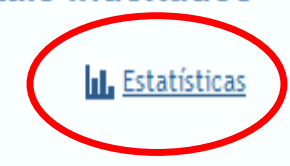

Percorrer

Para citar ou acessar este item utilize:

http://hdl.handle.net/10183/13293

Título Os usos pedagógicos dos vídeos digitais indexados

URI $\quad$ http://hdl.handle.net/10183/13293

Autor Dallacosta, Adriana

Orientador Tarouco, Liane Margarida Rockenbach

Co-orientador Franco, Sérgio Roberto Kieling

Data 2007

Nivel Doutorado

Todo o repositório

$\rightarrow$ Comunidades $\mathrm{C}$ colecões

$\rightarrow$ Títulos

$\rightarrow$ Autores

+ Palavras-chave

$\rightarrow$ Anos

Esta coleção

$\rightarrow$ Títulos

$\rightarrow$ Autores

$\rightarrow$ Palavras-chave

+ Anos

Meu cadastro

$\rightarrow$ Login UFRGS

$\rightarrow$ Login não UFRGS

Estatísticas

Instituição

Universidade Federal do Rio Grande do Sul. Faculdade de Educação. Programa de Pós-Graduação em Informática na Educação.

Itens mais

$\frac{\text { Itens mais }}{\text { baixados }}$

Construção do conhecimento

Indexação

Pedagogia de projetos

Sala de aula

Tecnologia de informação e comunicação

Video educativo 


\section{e) Os usos pedagógicos dos vídeos digitais indexados - Mozilla Firefox}

Arquivo É ditar Exibir Histórico Favoritos Ferramentas Ajūda

(4) C X \& $\square$ http://www.lume.ufrgs.br/handle/10183/13293/stats

औ. Google

2. Mais visitados Guia rápido Ulitimas notícias \& Google

Os usos pedagógicos dos vídeos digit...
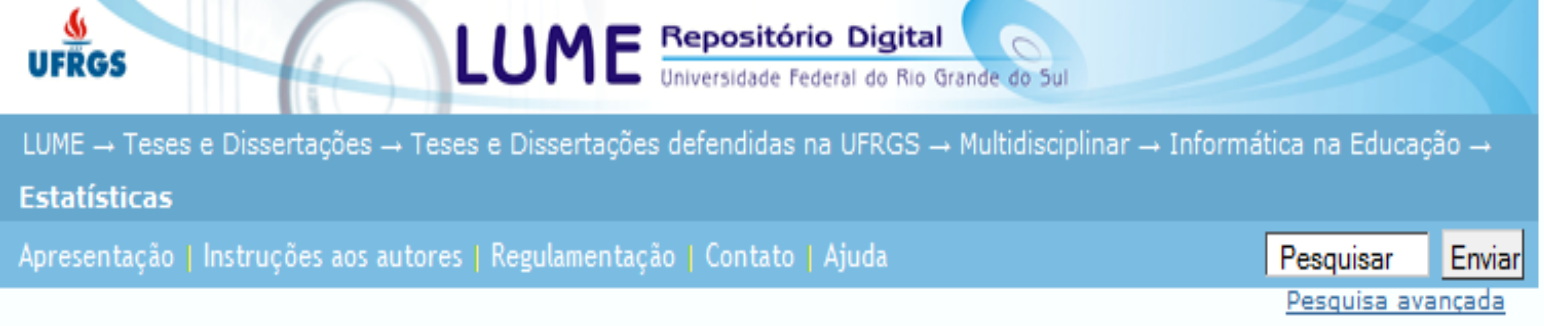

\section{Estatísticas}

Início da coleta: Jan/2008

\section{Os usos pedagógicos dos vídeos digitais indexados}

Estatísticas: por ano I por mês

Filtrar por data

\begin{tabular}{|c|c|c|c|}
\hline Data inicial & $\begin{array}{l}\text { Todos } \\
\text { Ano }\end{array}$ & $\begin{array}{l}\text { Todos V } \\
\text { Mês }\end{array}$ & $\begin{array}{l}\text { Todos } \\
\text { Dia }\end{array}$ \\
\hline Data final & $\begin{array}{l}\text { Todos }- \\
\text { Ano }\end{array}$ & $\begin{array}{l}\text { Todos - } \\
\text { Mâs }\end{array}$ & $\begin{array}{l}\text { Todos V } \\
\text { Dia }\end{array}$ \\
\hline
\end{tabular}

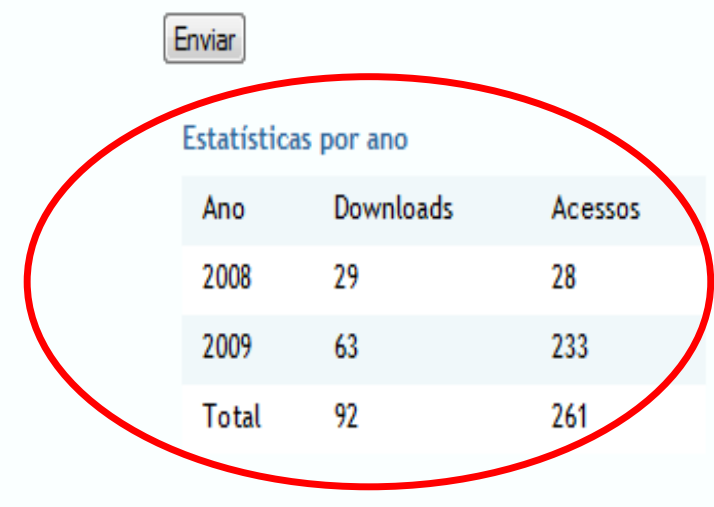

\section{Percorrer}

\section{Todo o repositório}

$\rightarrow$ Comunidades $e$ colecões

$\rightarrow \underline{\text { Títulos }}$

$\rightarrow$ Autores

$\rightarrow$ Palavras-chave

$\rightarrow$ Anos

\section{Esta coleção}

$\rightarrow \underline{\text { Títulos }}$

$\rightarrow$ Autores

$\rightarrow$ Palavras-chave

$\rightarrow$ Anos

Meu cadastro

$\rightarrow$ Login UFRGS

$\rightarrow$ Login não UFRGS

Estatísticas

$\rightarrow$ Downloads

$\rightarrow$ Itens mais baixados 


\begin{tabular}{|c|c|c|c|}
\hline Pais & Downloads & Pais & Acessos \\
\hline Brasil & 86 & Brasil & 245 \\
\hline (9) Portugal & 3 & 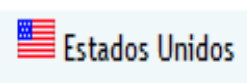 & 9 \\
\hline UFRGS* & 2 & UFRGS* & 7 \\
\hline E-Espanha & 1 & (1) Portugal & 5 \\
\hline 集 Reino Unido & 1 & Espanha & 1 \\
\hline IRomênia & 1 & 齒 Reino Unido & 1 \\
\hline
\end{tabular}
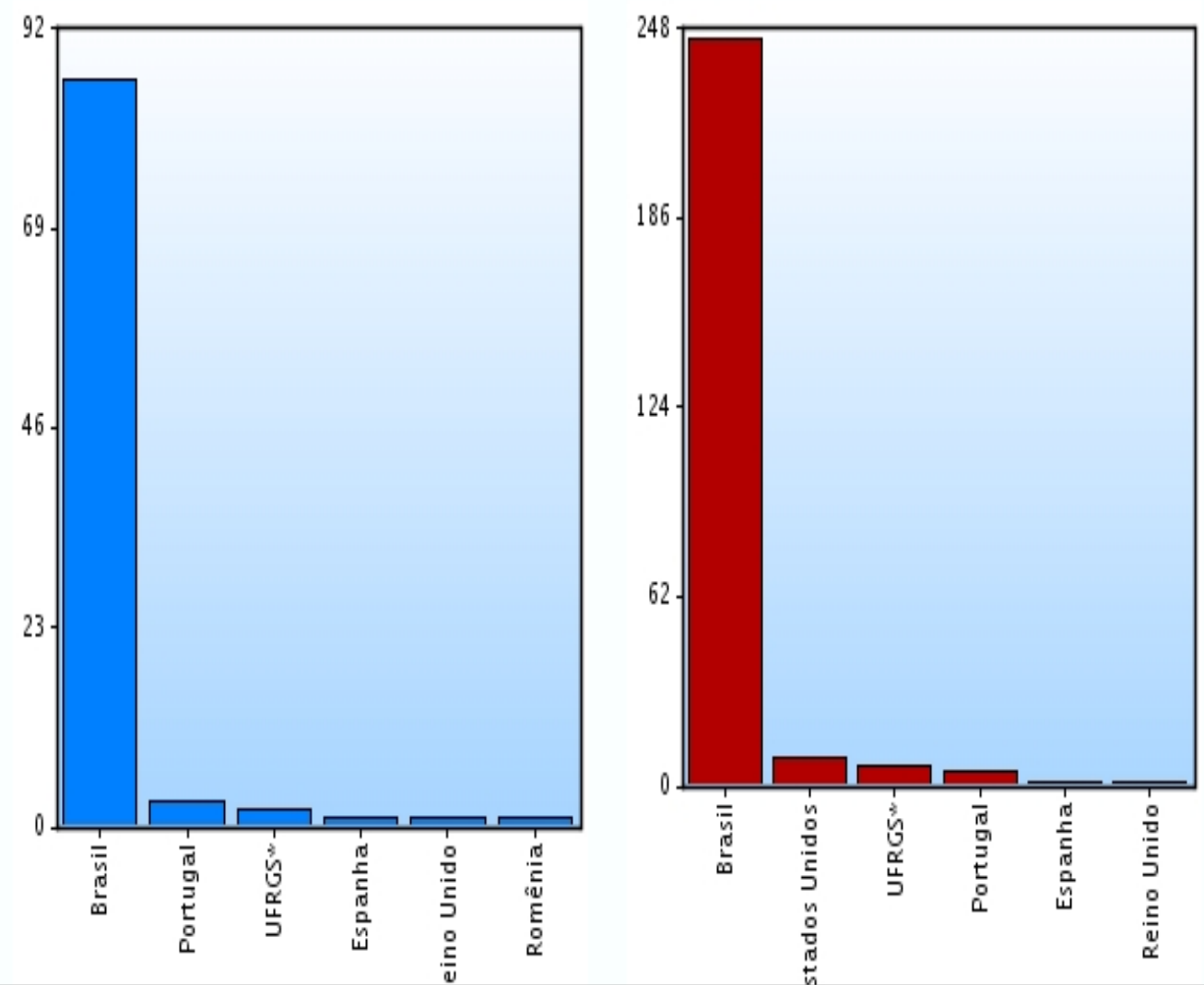


\section{A UFRGS \\ EM NÚMERO DE REGISTROS}

No mundo

$\Leftrightarrow 57^{\circ}$ lugar entre os 847 repositórios institucionais registrados

$\Leftrightarrow 30^{\circ}$ lugar entre os 327 repositórios institucionais que utilizam DSpace

Fonte: ROAR - Registry of Open Access Repositories, em 22 out. 2009. 


\section{A UFRGS}

\section{EM NÚMERO DE REGISTROS}

\section{No Brasil}

$\Leftrightarrow 6^{\circ}$ lugar entre os 70 repositórios registrados

$\Leftrightarrow 1^{\circ}$ lugar entre os 14 repositórios institucionais registrados, com 17.037 registros

$\Leftrightarrow 1^{\circ}$ lugar entre os 6 repositórios institucionais que utilizam DSpace

Fonte: ROAR - Registry of Open Access Repositories, em 22 out. 2009. 
ENDEREÇO

\section{http://www.lume.ufrgs.br}




\section{Obrigada!}

\section{bdigh-1@cpd.ufros.br

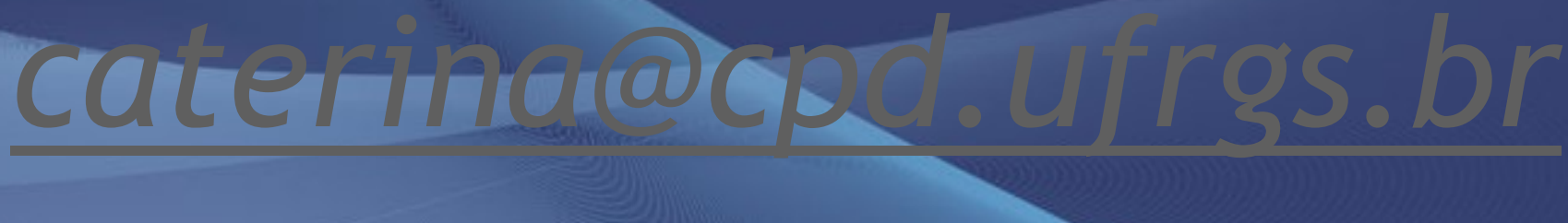

